\title{
Cartilage Endplate Thickness Variation Measured by Ultrashort Echo-Time MRI Is Associated With Adjacent Disc Degeneration
}

\author{
Britta Berg-Johansen, PhD, ${ }^{*} \dagger$ Misung Han, $\mathrm{PhD},{ }^{\ddagger}$ Aaron J. Fields, $\mathrm{PhD},{ }^{*}$ Ellen C. Liebenberg, BS, ${ }^{*}$ \\ Brandon J. Lim, BS, ${ }^{*}$ Peder EZ Larson, PhD, ${ }^{\dagger, \ddagger}$ Cigdem Gunduz-Demir, PhD,,${ }^{\$} \uparrow$ Galateia J. Kazakia, PhD, \\ Roland Krug, $\mathrm{PhD},{ }^{\ddagger}$ and Jeffrey C. Lotz, $\mathrm{PhD}^{*, \dagger}$
}

Study Design. A magnetic resonance imaging study of human cadaver spines.

Objective. To investigate associations between cartilage endplate (CEP) thickness and disc degeneration.

Summary of Background Data. Damage to the CEP is associated with spinal injury and back pain. However, CEP morphology and its association with disc degeneration have not been well characterized.

Methods. Ten lumbar motion segments with varying degrees of disc degeneration were harvested from six cadaveric spines and scanned with magnetic resonance imaging in the sagittal plane using a $\mathrm{T}_{2}$-weighted two-dimensional (2D) sequence, a threedimensional (3D) ultrashort echo-time (UTE) imaging sequence, and a 3D $\mathrm{T}_{1 \rho}$ mapping sequence. CEP thicknesses were calculated from 3D UTE image data using a custom, automated algorithm, and these values were validated against histology measurements. Pfirmann grades and $T_{1 \rho}$ values in the disc were assessed and correlated with CEP thickness.

Results. The mean CEP thickness calculated from UTE images was $0.74 \pm 0.04 \mathrm{~mm}$. Statistical comparisons between histology and UTE-derived measurements of CEP thickness showed significant

From the *Department of Orthopaedic Surgery, University of California, San Francisco, CA; ${ }^{\dagger}$ Department of Bioengineering, UC Berkeley/UCSF Joint Program in Bioengineering, Berkeley, CA; ${ }^{\ddagger}$ Department of Radiology and Biomedical Imaging, University of California, San Francisco, CA; ${ }^{\S}$ Department of Computer Engineering, Bilkent University, Ankara, Turkey; and Neuroscience Graduate Program, Bilkent University, Ankara, Turkey. Acknowledgment date: May 25, 2017. First revision date: August 8, 2017. Acceptance date: September 6, 2017.

BB-J, MH, and AJF contributed equally to this work.

The manuscript submitted does not contain information about medical device(s)/drug(s).

National Institutes of Health (AR063705) funds were received in support of this work.

Relevant financial activities outside the submitted work: grants.

Address correspondence and reprint requests to Jeffrey C. Lotz, PhD, University of California, 513 Parnassus Avenue, S-1157, San Francisco, CA 94143-0514; E-mail: jeffrey.lotz@ucsf.edu

DOI: 10.1097/BRS.0000000000002432 agreement, with the mean difference not significantly different from zero $(P=0.32)$. Within-disc variation of $\mathrm{T}_{1 \rho}$ (standard deviation) was significantly lower for Pfirmann grade 4 than Pfirrmann grade $3(P<0.05)$. Within-disc variation of $\mathrm{T}_{1 \rho}$ and adjacent CEP thickness heterogeneity (coefficient of variation) had a significant negative correlation $(r=-0.65, P=0.04)$. The standard deviation of $\mathrm{T}_{1 \rho}$ and the mean CEP thickness showed a moderate positive correlation $(r=0.40, P=0.26)$.

Conclusion. This study demonstrates that quantitative measurements of CEP thickness measured from UTE magnetic resonance imaging are associated with disc degeneration. Our results suggest that variability in CEP thickness and $T_{1 \rho}$, rather than their mean values, may serve as valuable diagnostic markers for disc degeneration.

Key words: cartilage endplate, disc degeneration, endplate thickness, image processing algorithm, image segmentation, intervertebral disc, low back pain, lumbar spine, Pfirrmann grade, spatial variation, T1rho, ultrashort echo-time MRI (UTE MRI).

\section{Level of Evidence: N/A}

Spine 2018;43:E592-E600

ow back pain is the leading cause of disability worldwide, affecting $70 \%$ to $85 \%$ of the population during their lives. ${ }^{1}$ Costs related to back pain are estimated to exceed $\$ 100$ billion every year in the United States. ${ }^{2} \mathrm{With}$ increasing average life expectancy, the prevalence of low back pain is likely to increase. ${ }^{3}$ While the precise causes of low back pain are not well understood, intervertebral disc degeneration is believed to be a leading factor. ${ }^{4,5}$

The intervertebral disc is avascular and is composed of a central gel-like nucleus pulposus, a surrounding annulus fibrosus, and superiorly and inferiorly located cartilage endplates (CEPs). ${ }^{6}$ The nucleus pulposus mainly consists of proteoglycan and water so as to resist spinal compression hydrostatically. The annulus is mainly composed of type I collagen and forms a firm but flexible outer layer that provides strength and flexibility. The CEP is a 0.1 to 
$1.6 \mathrm{~mm}$ layer of hyaline cartilage between the vertebra and central portion of the disc (nucleus and inner annulus)., The CEP resists intradiscal pressure and allows nutrient transport by diffusion into the disc from vertebral blood vessels. ${ }^{7,9}$

Early stages of disc degeneration include biochemical changes such as a loss of proteoglycans and hydration. ${ }^{10}$ In later stages, morphologic changes occur, including disc height loss, nucleus pulposus herniation, and annular tears. ${ }^{11}$ With aging, the CEP becomes calcified and less permeable,${ }^{12,13}$ and consequently, nutrient transport across the endplates decreases with age, injury, and degeneration. ${ }^{14,15}$ Damage to the CEP also associates with spinal injury and an increased risk of back pain. ${ }^{16-19}$ Despite the potentially important role of the CEP in disc degeneration and back pain/injury, CEP morphology and its association with disc degeneration have not been well characterized.

One reason for this lack of characterization is that the CEP has a short $T_{2}$ relaxation (spin-spin relaxation) time, and standard magnetic resonance imaging (MRI) sequences are unable to capture its signal. However, recent studies have demonstrated the feasibility of using a short-TE or ultrashort TE (UTE) MRI sequence for depicting the morphology of the CEP and quantifying the thickness. ${ }^{20-23}$ Disc degeneration is often measured with the semiquantitative Pfirmann grading method, ${ }^{24}$ and has also been measured by $\mathrm{T}_{2}$ and $\mathrm{T}_{1 \mathrm{p}}$ relaxation (spin-lattice relaxation in the rotating frame) times. $T_{2}$ relaxation time is sensitive to changes in collagen and water, ${ }^{25,26}$ while $T_{1 \rho}$ relaxation time is also sensitive to macromolecules and has the potential to identify early biochemical changes in the disc..$^{27-29}$

The objective of this study was to evaluate whether UTE MRI-derived CEP thickness measurements can be made accurately, and associate with disc degeneration. This association is theoretically plausible since CEP thickness can alter nutrient transport into the disc and thereby affect disc matrix quality (represented by $\mathrm{T}_{1 \rho}$ ). In our first aim, we measured the CEP thickness from 3D UTE images using a novel automatic thresholding algorithm, and validated the algorithm with histology-derived measurements. In our second aim, we measured disc $\mathrm{T}_{1 \rho}$ relaxation times and compared these with CEP thickness measurements.
Correlations between MRI-derived CEP thickness values, Pfirrmann grades, and disc $T_{1 \rho}$ were assessed to investigate the usefulness of CEP thickness measurement as a diagnostic tool.

\section{METHODS}

\section{Image Acquisition}

Ten lumbar motion segments (4 L1-L2, 2 L2-L3, 3 L4-L5, 1 L5-S1) with varying degrees of disc degeneration were harvested from six human cadaver spines (age: $58.5 \pm 5.2$ yr; 4 males, 2 females) within 1 week postmortem and scanned with MRI. Each motion segment was attached to a rigid alignment guide that served as a fiducial marker for aligning UTE MRI and histology locations (Figure 1A-E). The MRI-compatible alignment guide is constructed of acrylic, and the plastic mounting piece (Figure 1A, B) leaves a $13 \times 13 \mathrm{~mm}$ square channel (Figure 1C). MRI was conducted using a Discovery MR 750W 3T scanner (GE Healthcare, Waukesha, WI) and an eight-channel phasedarray wrist coil (Invivo, Gainesville, FL). The imaging protocol included a $\mathrm{T}_{2}$-weighted $2 \mathrm{D}$ fast spin-echo (FSE) sequence, a $3 \mathrm{D}$ UTE imaging sequence, and a $3 \mathrm{D} \mathrm{T}_{1 \rho}$ mapping sequence. For all three sequences, imaging was performed in the sagittal plane.

$\mathrm{T}_{2}$-weighted 2D FSE sequence used a repetition time (TR) of 7000 to $8000 \mathrm{~ms}$, TE of 85 to $90 \mathrm{~ms}, 8 \times 8 \mathrm{~cm}^{2}$ field of view, $256 \times 192$ matrix size, $1.5 \mathrm{~mm}$ slice thickness, and 36 to 44 slices. UTE imaging used 3D radial acquisition combined with nonselective excitation, and scan parameters included $75 \mu \mathrm{s} \mathrm{TE}, 15^{\circ}$ flip angle, $12 \mathrm{~ms}$ TR, $8 \times 8 \times$ $7 \mathrm{~cm}^{3}$ field of view, $0.5 \times 0.5 \times 1.5 \mathrm{~mm}^{3}$ spatial resolution, and 40 to 48 slices. Fat suppression was applied every five radial spoke acquisitions to minimize off-resonance artifacts from fatty components in the vertebral bodies. Magnetization-prepared angle-modulated partitioned-k-space spoiled gradient echo snapshots (3D MAPSS) ${ }^{30}$ with eight spin-lock times $(0,2,4,8,12,20,40$, and $80 \mathrm{~ms})$ and $500 \mathrm{~Hz}$ spin-lock frequency used for $T_{1 \rho}$ mapping in the disc, with imaging parameters of $10 \times 10 \mathrm{~cm}^{2}$ field of view, $256 \times 128$ matrix size, $4 \mathrm{~mm}$ slice thickness, and 14 to 18 slices. On the same day of MR imaging, motion segments were scanned with

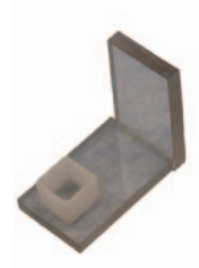

A

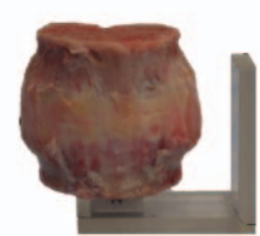

B

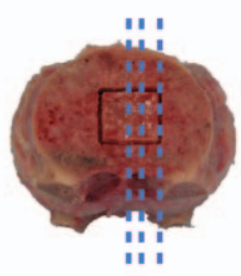

C

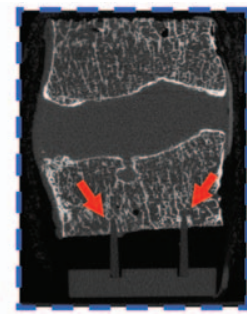

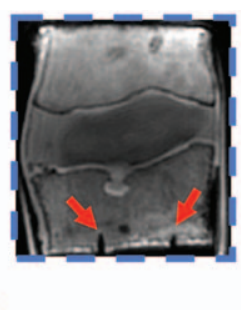

$E$

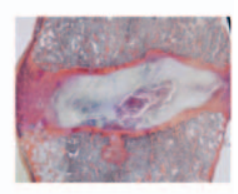

$\mathrm{F}$

Figure 1. Method for coaligning histology and MR images. (A, B) Motion segment is placed on rigid alignment guide with $13 \times 13 \mathrm{~mm}$ mounting square; $(\mathbf{C})$ histology, HR-pQCT, and MR images are taken parallel to alignment channels of mounting square to ensure images are coplanar (blue lines denote parallel image planes); matching sagittal (D) HR-pQCT, (E) MRI, and (F) histology slices are determined relative to location of channel (red arrows). Visible channels in (D) and (E) indicate that this slice is within the square grid of the alignment guide. HRPQCT indicates high-resolution peripheral quantitative computed tomography; MR, magnetic resonance; MRI, magnetic resonance imaging. 
high-resolution peripheral quantitative computed tomography (HR-pQCT) with an XtremeCT scanner (Scanco, Medical AG, Bruttisellen, Switzerland), (providing $41 \mu \mathrm{m}$ voxel size) to acquire more detailed structures of our motion segments.

\section{Histology}

After imaging, motion segments were sectioned into 5 to $10 \mathrm{~mm}$ thick para-sagittal slabs for histology. Slabs were sectioned parallel to the channels of the alignment guide (Figure 1C) to ensure sections were coplanar with UTE. Two para-sagittal slabs from each motion segment were chosen based on known regions of CEP defects and/or Modic regions from MRI. Slabs were fixed, decalcified with ethylenediaminetetraacetic acid, and sectioned ( $7 \mu \mathrm{m}$ thickness). Sections were stained with a trichrome stain containing aniline blue, orange $G$, and acid fuchsin. For subsequent validation of UTE CEP thickness measurements, CEP thickness was measured on photomicrographs using image analysis software (ImageJ, NIH, Bethesda, MD) at 50 evenly spaced positions from anterior to posterior.

\section{UTE MRI CEP Thickness Measurement and Validation With Histology}

UTE images were interpolated to a voxel size of $0.25 \times 0.25$ $\times 0.5 \mathrm{~mm}^{3}$. Images were then processed using a custom, automated algorithm in Matlab (Mathworks, Natwick, MA) to calculate CEP thickness. The algorithm loops through the following steps for each MRI slice for all motion segments (Figure 2):

(1) Contours the endplates;

(2) Dilates the contours, and masks dilated contours over original image;

(3) Rebins the data using Lanczos interpolation ${ }^{31,32}$ to quadruple the number of pixels (thereby enhancing apparent resolution);

(4) Identifies and crops the inferior and superior endplate regions;
(5) Thresholds each endplate separately using adaptive (region-dependent) thresholding, in which a unique threshold is calculated for different regions across the endplate with varying image intensity and contrast. Otsu thresholding $^{33}$ is used to select each region's threshold using the discriminant criterion, which maximizes the separability of the resultant classes in gray levels;

(6) Calculates thickness across the endplate, perpendicular to the tangent line at each $\mathrm{x}$-location along the bottom of the endplate;

(7) Smooths the data using a one-dimensional digital filter.

This algorithm is fully automatic besides the addition of a "threshold multiplier" (ranged from 1.1 to 1.35) as an input to certain specimens to tweak each region's threshold by the multiplier value.

Thickness measurements from UTE images were then validated with histology measurements. The matching MRI slice for each histology section was determined using the novel coalignment method outlined in Figure 1, which uses the channels from the alignment guide as fiducial markers. In particular, the location of the sagittal histology section was first determined using the HR-pQCT images and then the matching MRI slice was determined by checking the distance to denoted channels on UTE images. Altogether, $\mathrm{n}=21$ individual UTE MRI slices from five motion segments were matched to histology sections, yielding $n=40$ matched CEPs (inferior and superior for each slice). For each CEP, thickness measurements were compared between UTE images and histology at each of the 50 evenly spaced anterior-posterior positions.

Several comparison metrics were used to evaluate the agreement between UTE MRI-derived estimates of CEP thickness and their respective site-matched histology measurements. For UTE-histology pair, the systematic bias and noise in the thickness estimates were determined by calculating the mean difference and root-mean-square deviation (RMSD) for the 50 evenly spaced anterior-posterior
1. Loop by slice

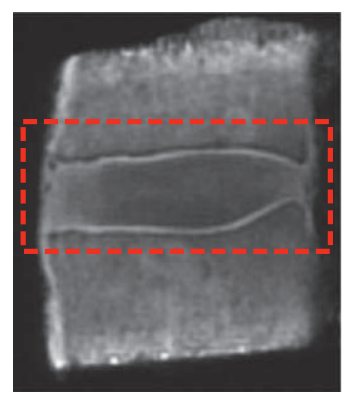

2. Contour and mask

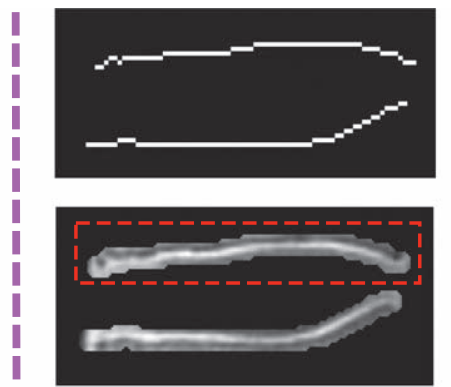

3. Re-bin, threshold, and measure thickness

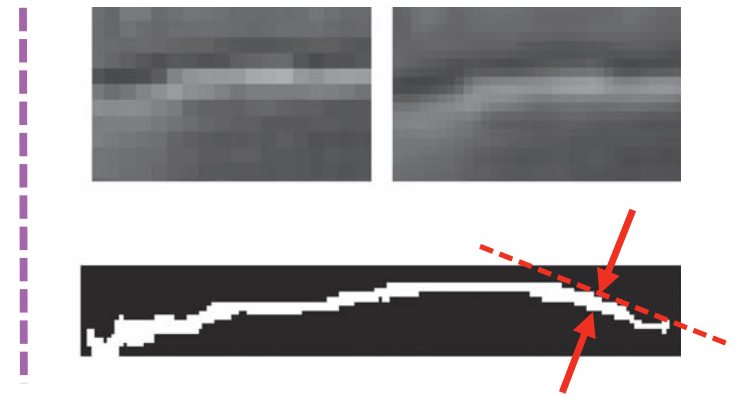

Figure 2. Steps of UTE CEP thickness algorithm. (1) Algorithm loops through MRI slices, (2) contours and masks the endplates, (3) rebins to increase resolution (top left image is original, top right image is rebinned), then thresholds the endplate and calculates CEP thickness values (red arrows) that are perpendicular to the tangent line (red dotted line) at each horizontal location. Panel 2 shows boxed region in panel 1 , and panel 3 shows images for top endplate (dashed box in panel 2). CEP indicates cartilage endplate; MRI, magnetic resonance imaging; UTE, ultrashort echo-time. 
positions. The proportional bias was determined from Bland-Altman plots for each endplate. General agreement between the two measurement techniques was assessed using correlation analysis.

To evaluate possible tissue shrinking effects caused by histology, we compared the distance between features on serial histologic sections to their distance measured in the HRpQCT scans (acquired before histologic processing). For example, if 35 serial histologic sections ( $7 \mu \mathrm{m}$ each) separated two trabecular structures, their relative separation $(35 \times$ $7 \mu \mathrm{m}=245 \mu \mathrm{m})$ can be compared with their relative separation on the HR-pQCT datasets (41 $\mu$ m slice thickness).

\section{MR Image Assessment and Association With Degeneration}

Disc degeneration level was assessed using the Pfirrmann scoring system ${ }^{24}$ based on $\mathrm{T}_{2}$-weighted FSE images by a radiologist with 25 years of experience. The Pfirrmann grading scheme classifies disc degeneration from Grades 1 to 5 (Grade $1=$ nondegenerated; Grade $5=$ severely degenerated). Table 1 summarizes the donor age/sex and level of each motion segment, along with the associated Pfirrmann grade.

$\mathrm{T}_{1 \rho}$ values in the disc were calculated using the MAPSS images with the eight different spin-lock times. The signal intensity at a certain spin-lock time (TSL) can be written as $\mathrm{S}_{\text {TSL }}=\mathrm{S}_{\mathrm{O}} \exp \left(-\frac{\mathrm{TSL}}{T_{1 \mathrm{p}}}\right)$, where $\mathrm{S}_{0}$ is the signal intensity at $\mathrm{TSL}=0$. Therefore, monoexponential fitting on a pixel-bypixel basis was performed using the Levenberg-Marquardt algorithm. Manual segmentation of the disc was performed using the in-house image processing toolkit ${ }^{34}$ based on the MAPSS images with a spin lock time of $0 \mathrm{~ms}$. Because of limited spatial resolution and dark signal of the CEPs from the MAPPS images, $T_{1 \rho}$ in the CEP was not quantifiable. Nucleus pulposus regions of the discs were segmented and estimated as the central half-width regions of the discs (along the anterior to posterior direction). It has been shown that the CEP regions above and below the nucleus pulposus are permeable and allow for nutrient diffusion into the disc. ${ }^{35} \mathrm{CEP}$ thickness values neighboring these segmented disc regions were used for correlation analyses.

\begin{tabular}{|l|c|c|c|}
\hline \multicolumn{4}{|c|}{ TABLE 1. Summary of Sample Sets } \\
\hline $\begin{array}{l}\text { Motion } \\
\text { Segment }\end{array}$ & Subject & $\begin{array}{c}\text { Disc } \\
\text { Level }\end{array}$ & $\begin{array}{c}\text { Pfirrmann } \\
\text { Grade }\end{array}$ \\
\hline 1 & 57 years (male) & L1-L2 & 2 \\
\hline 2 & 57 years (male) & L4-L5 & 3 \\
\hline 3 & 65 years (male) & L1-L2 & 3 \\
\hline 4 & 65 years (male) & L4-L5 & 3 \\
\hline 5 & 57 years (female) & L1-L2 & 3 \\
\hline 6 & 57 years (female) & L2-L3 & 3 \\
\hline 7 & 49 years (male) & L1-L2 & 3 \\
\hline 8 & 63 years (male) & L2-L3 & 4 \\
\hline 9 & 60 years (male) & L4-L5 & 4 \\
\hline 10 & 60 years (male) & L5-S1 & 4 \\
\hline
\end{tabular}

For each segment, the mean and coefficient of variation (CV) of the CEP thickness, averaged over the segmented superior and inferior CEP regions, were calculated as a possible degeneration biomarker. Two-tailed $t$ tests were conducted to assess differences in mean CEP thickness, CV CEP thickness, and mean and standard deviation of $T_{1 \rho}$ between Pfirrmann grades 3 and 4. Only one disc had Pfirrmann grade 2, and thus Pfirmann grade 2 was not included in $t$ tests. Univariate linear regression analysis was used to assess correlations between disc properties (mean $\mathrm{T}_{1 \rho}$, standard deviation of $\mathrm{T}_{1 \rho}$, and Pfirrmann grade) and CEP properties (mean CEP thickness and CV CEP thickness), and Pearson correlation coefficients were calculated between the mean and standard deviation of $T_{1 \rho}$ and the mean and CV CEP thickness. Significance of statistical tests was defined as $P<0.05$.

\section{RESULTS}

\section{Validation and Results of UTE Thickness Algorithm}

Thickness maps from both superior and inferior CEPs for all 10 motion segments demonstrated significant heterogeneity (Figure 3). The mean CEP thickness was $0.74 \pm 0.04 \mathrm{~mm}$, not much different from the reported values $(0.62 \pm 0.29 \mathrm{~mm}) .^{7}$ There was no difference in mean thickness values between superior and inferior CEPs $(P=0.46)$. CEPs tended to be thicker around the periphery and thinner in the central portion. Statistical comparison between histology and UTE-MRI-derived measurements of CEP thickness showed significant agreement (Figure 4A-D). Although UTE MRI over-estimated CEP thickness (mean difference $=0.02 \pm 0.13 \mathrm{~mm}$ ), this over-estimation was not significantly different from zero $(P=0.32)$. UTE MRI had a small but significant proportional bias $(-0.25 \pm 0.73 \mathrm{~mm}$, $P=0.03$ ). This negative proportional bias indicated that UTE MRI's over-prediction of thickness was lesser in locations where the CEP was thicker. Histology and UTE MRIbased measurements of CEP thickness variation were significantly correlated for 28/40 CEP sections. The mean regression slope was $0.35 \pm 0.27$, indicating that UTE-predicted variations in thickness were generally greater in magnitude than histology-measured variations in thickness. The random noise (RMSD) of the UTE MRI measurements was $0.23 \pm 0.06 \mathrm{~mm}$. Comparisons of trabecular geometry between histology and HR-pQCT scans showed that shrinking of the bony tissue was less than $5 \%$ in three motion segment slabs: $3.4 \%, 4.5 \%$, and $2.4 \%$. Based on this small amount of shrinking in the bone, and given that our measurements of CEP thickness are within the range measured by photomicrographs $(0.62 \pm 0.29 \mathrm{~mm}),{ }^{7}$ we did not adjust our histologic thickness measurements.

\section{Correlations Between CEP Thickness and Disc Degeneration}

UTE-derived CEP thickness maps and disc $T_{1 \rho}$ maps varied with Pfirrmann grade, with degenerated discs exhibiting poorly delineated CEPs with point defects and lower 


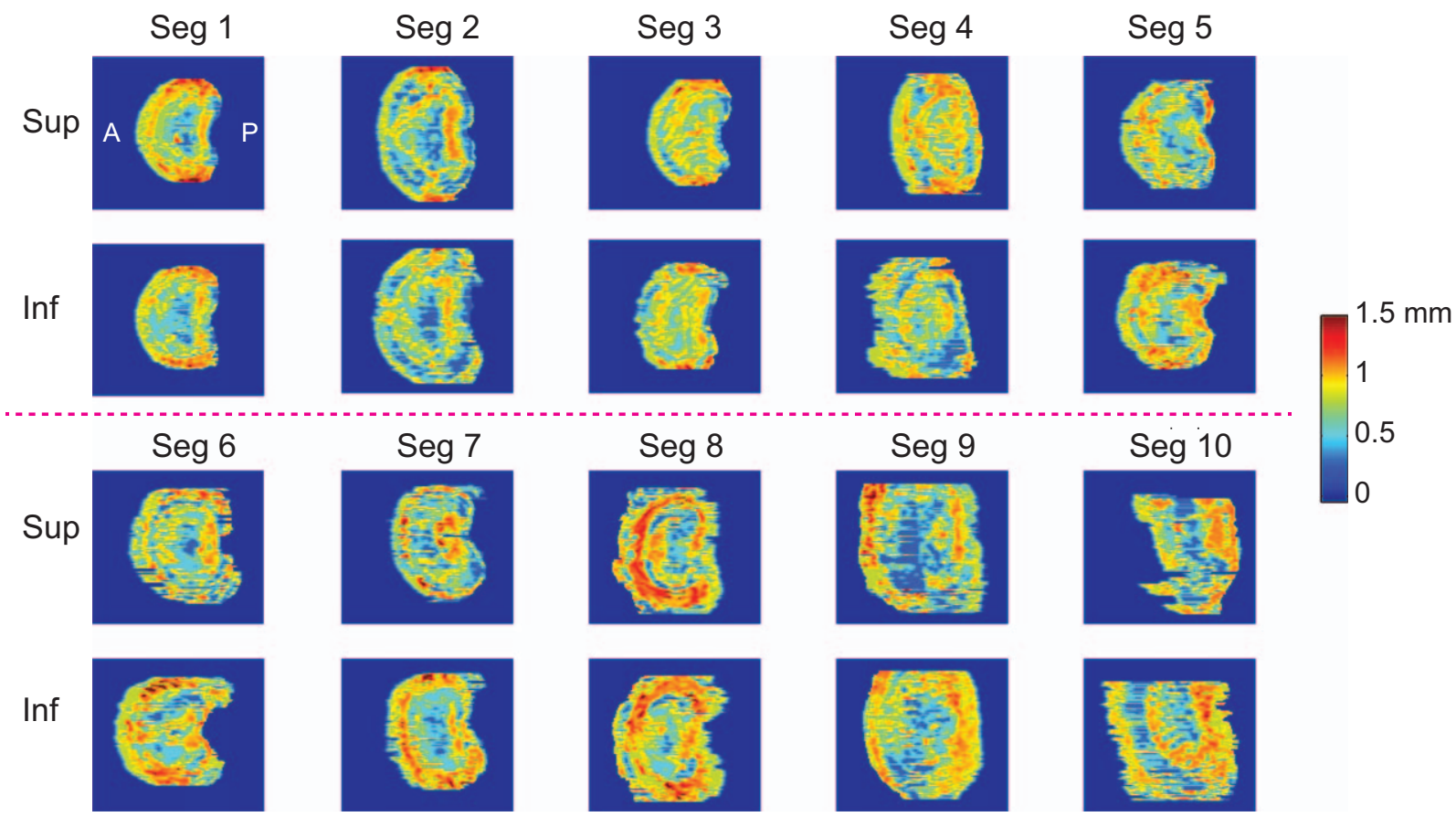

Figure 3. CEP thickness maps measured using Matlab algorithm on UTE images from all 10 motion segments. The anterior-posterior direction is from the left to the right of the maps. CEP indicates cartilage endplate.

thickness values (Figure 5A-H). Several statistical trends were observed between the Pfirrmann grade, CEP thickness from UTE images, and disc $\mathrm{T}_{1 \rho}$ (Figure 6A-D). Even with a limited sample size, the standard deviation of $T_{1 \rho}$ was significantly lower for Pfirrmann grade 4 than Pfirrmann grade $3(P<0.05)$. The mean $\mathrm{T}_{1 \rho}$ also decreased with Pfirrmann grade, although the difference was not significant. The CV CEP thickness was significantly higher for
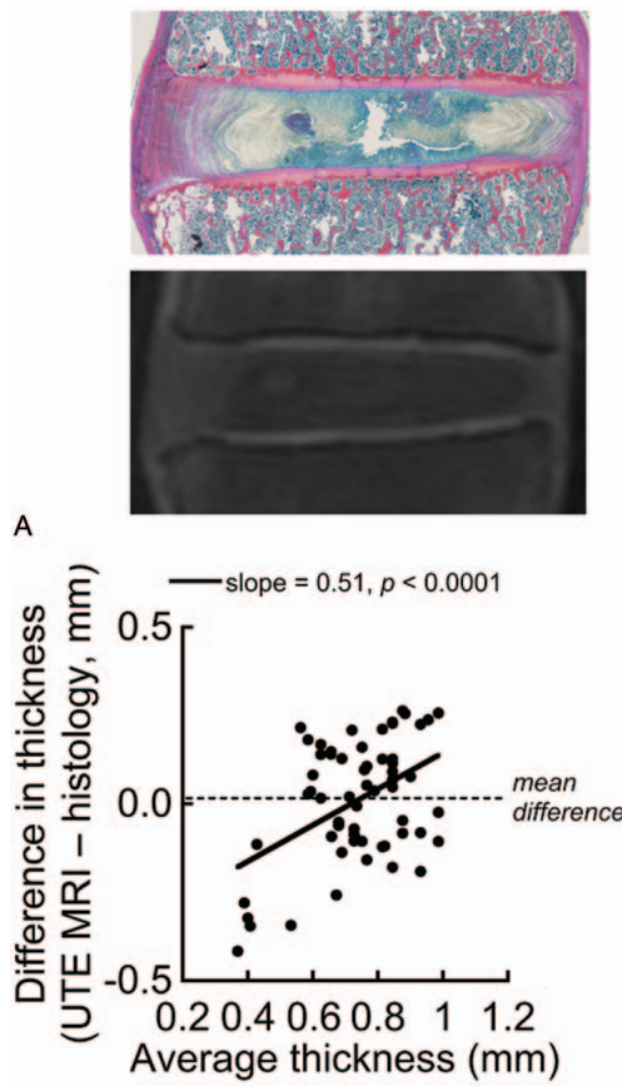

C
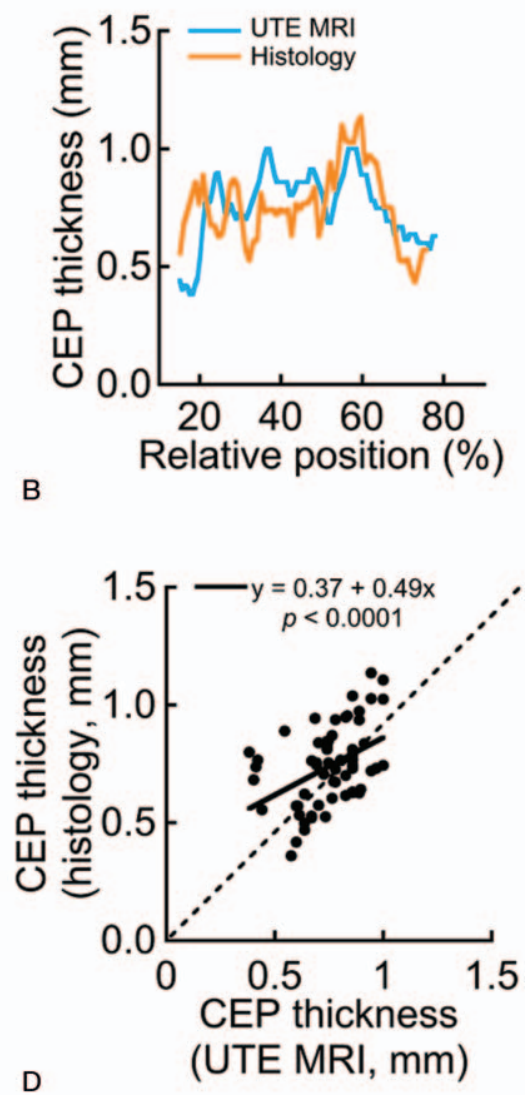

Figure 4. (A) Site-matched histology and UTE MR images. (B) Comparison of CEP thickness between UTE MRI and histology for a selected CEP section. (C) Bland-Altman plot for the same section, where proportional bias (slope) is 0.51 and mean difference is $0.013 \mathrm{~mm}$. (D) Correlation between UTE and histology for the same section (slope $=0.37$, which is less than 1 , indicating that UTE overestimated the variation in endplate thickness). Dashed line denotes identical thickness measurement between UTE and histology. CEP indicates cartilage endplate; MR, magnetic resonance; MRI, magnetic resonance imaging; UTE, ultrashort echo-time. 
UTE image

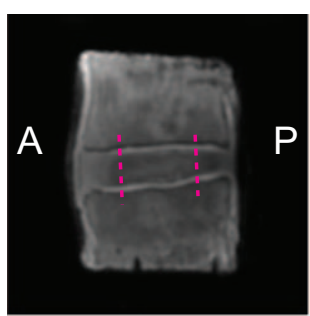$$
\text { Motic }
$$

A

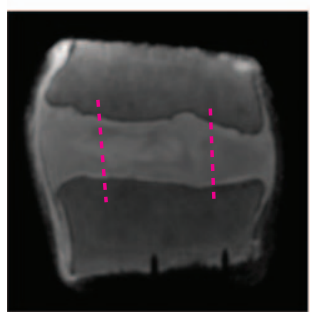

Motion segment 2 (grade 4)

E
$\mathrm{T}_{1 \rho}$ map

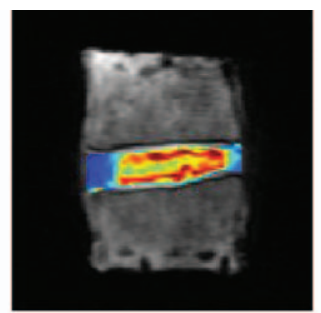

B

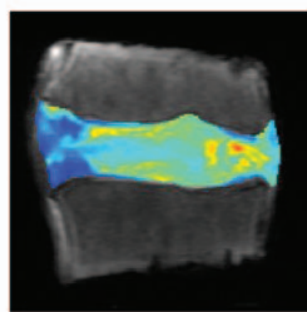

F
Superior CEP thickness map

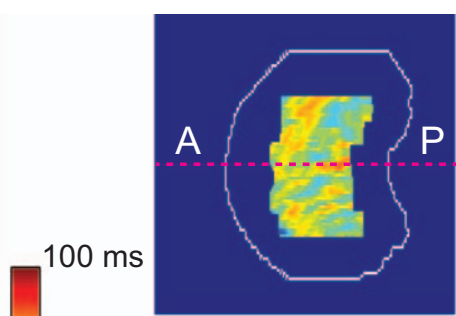

C

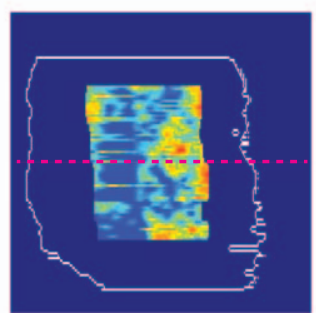

G
Inferior CEP

thickness map

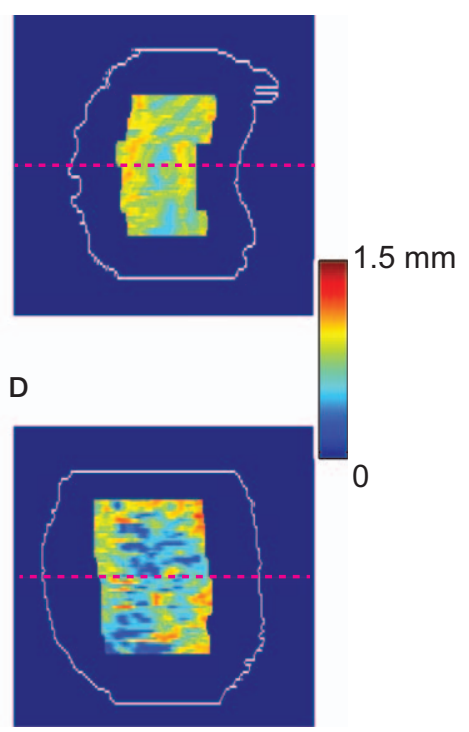

$\mathrm{H}$

Figure 5. (A, E) UTE images of motion segments with Pfirrmann Grade 3 and 4. Pfirrmann Grade 3 disc has well-delineated CEPs with high and homogeneous thickness values, while Pfirrmann Grade 4 disc has poorly delineated CEPs with point defects and lower thickness values. The region of the central half width of the disc is denoted with two pink dashed lines. The anterior-posterior direction is from the left to right. (B, F) $\mathrm{T}_{1 \rho}$ maps in the discs. (C, D, G, H) Thickness maps of CEPs are located superior and inferior to the segmented discs. The white contours represent the overall CEP boundaries before segmentation. The slice locations for (A) and (E) are denoted by pink dashed lines. CEP indicates cartilage endplate; UTE, ultrashort echo-time.

Pfirrmann grade 4 than Pfirrmann grade $3(P<0.05)$, while the mean CEP thickness was unaffected by Pfirrmann grade.

Linear regression (Figure 7A-D) showed that the standard deviation of $\mathrm{T}_{1 \rho}$ and CV CEP thickness had a significant negative correlation $(\mathrm{r}=-0.65, P=0.04)$. The mean $\mathrm{T}_{1 \rho}$ and CV CEP thickness also had a moderate negative correlation, but this correlation was not significant $(\mathrm{r}=-0.36, P=0.31)$. The standard deviation of $\mathrm{T}_{1 \rho}$ and the mean CEP thickness showed a moderate positive correlation $(\mathrm{r}=0.40, P=0.26)$, while the mean CEP thickness and mean $\mathrm{T}_{1 \rho}$ showed no correlation $(\mathrm{r}=0.11$, $P=0.76)$.

\section{DISCUSSION}

In this study, we investigated whether quantitative measurements of CEP morphology could be made on excised motion segments using UTE MRI, and whether these measurements have clinical utility. MR imaging was performed on a clinical scanner, and thus in vivo application can be possible with imaging parameter and coil adaptations. Even with limited samples with mainly Pfirrmann grades of 3 and 4, we observed that the variability in both CEP thickness and $T_{1 \rho}$ was significantly related to degeneration. Specifically, CV CEP thickness increased with the Pfirrmann grade, probably because CEP defects and resultant local thinning would increase $C V$ values, while the standard deviation of $T_{1 \rho}$

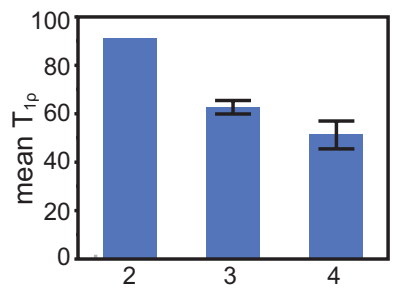

A

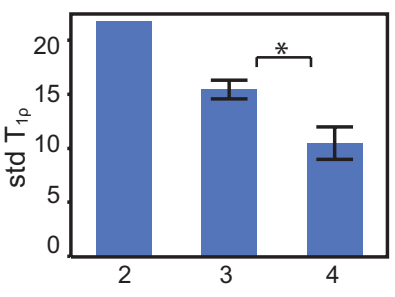

B

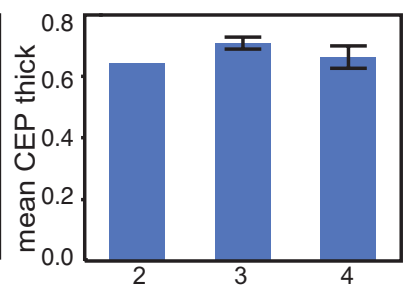

C

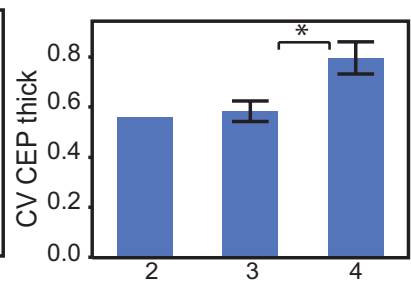

D

Pfirrmann grade

Figure 6. (A-D) The mean and standard deviation of $T_{1 \rho}$ in the segmented disc and the mean and CV of CEP thickness averaged between the superior and inferior maps as a function of the Pfirrmann grade. Notably, the standard deviation of $\mathrm{T}_{1 \rho}$ is significantly lower for Pfirrmann grade 4 than 3 (B), and the CV CEP thickness is significantly higher for Pfirrmann grade 4 than 3 (D). Error bars represent \pm standard error. ${ }^{*}$ indicates significant difference in the two-sample $t$ test $(P<0.05)$. CEP indicates cartilage endplate; CV, coefficient of variation. 

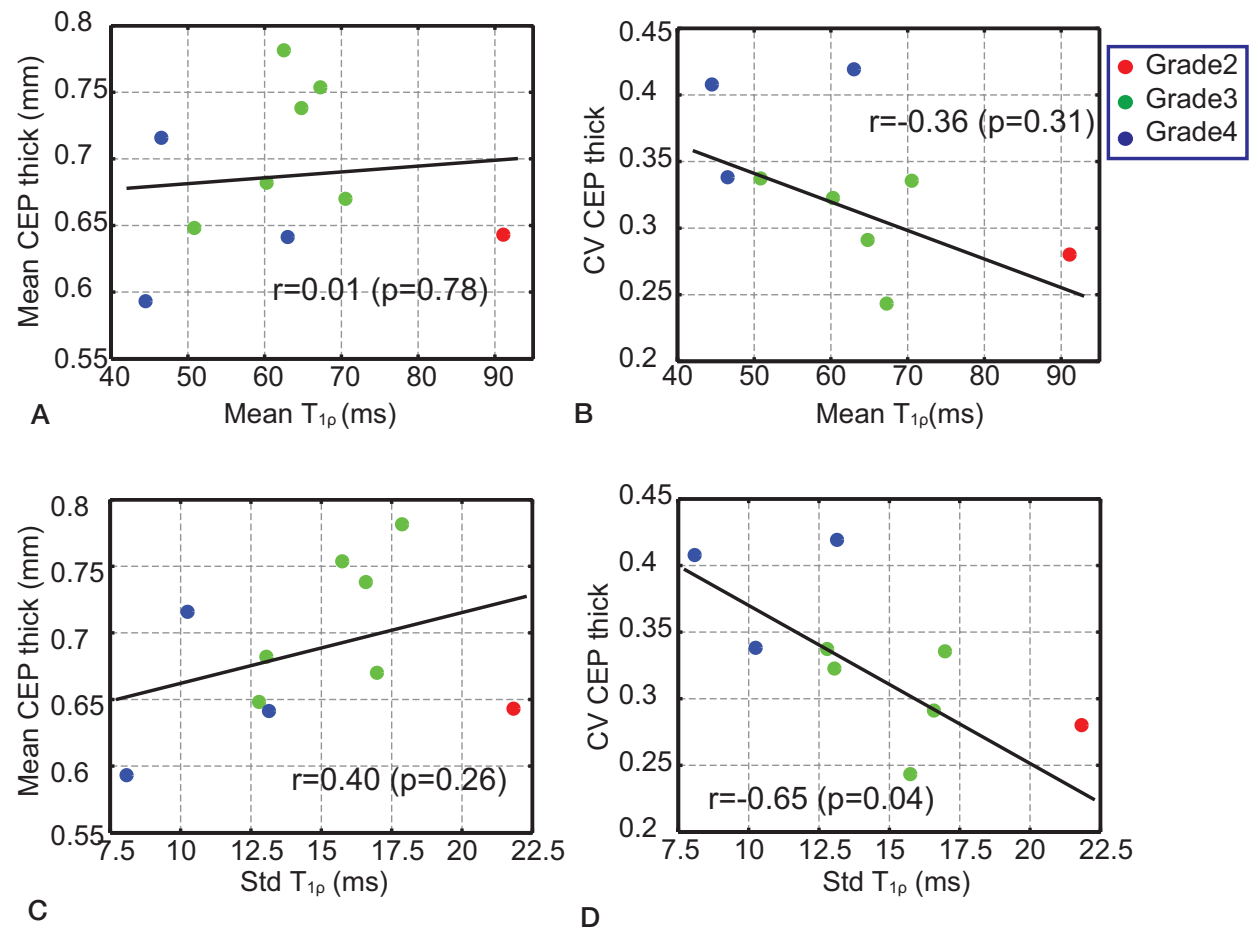

D

Figure 7. (A-D) Linear regression of the mean and $\mathrm{CV}$ of CEP thickness versus the mean and standard deviation of $\mathrm{T}_{1 \rho}$. The standard deviation of $\mathrm{T}_{1 \rho}$ and the CV CEP thickness have a significant negative correlation (D). Data points are color-coded by Pfirrmann grade. Pearson correlation coefficients and $P$ values are also denoted. CEP indicates cartilage endplate; $\mathrm{CV}$, coefficient of variation.

decreased with the Pfirrmann grade. However, the mean values of both CEP thickness and $T_{1 \rho}$ were not significantly associated with Pfirrmann grade or with each other. Taken together, these findings indicate that spatial heterogeneity in CEP and disc properties are more indicative of degeneration than the more commonly reported mean values of these properties. Apart from this, we also assessed correlation between the mean and CV CEP thickness and disc height, but no significant correlation was observed.

While several studies have reported calcification of the cartilage endplates with degeneration, ${ }^{14,15}$ only two studies have quantified human CEP thickness using MRI, ${ }^{20,22}$ one of which attempted to correlate CEP thickness with degeneration. These studies used a 3D gradient-echo sequence with a $3.7 \mathrm{~ms}$ TE, and calculated CEP thickness by manually outlining the CEP from several slices in ImageJ. No correlations were observed between CEP thickness and degeneration (as assessed by $T_{2}$ relaxation time and Pfirrmann grade). However, the authors did not assess heterogeneity in CEP thickness, which our findings suggest may be a more significant indicator of disc degeneration.

We present the first automatic, histologically validated technique for quantitatively measuring CEP thickness in three dimensions using MR images. While other studies have quantified CEP thickness manually and discretely using $2 \mathrm{D}$ slices, ${ }^{20,22,36}$ no algorithms to date have measured CEP thickness continuously across the entire endplate volume. To maximize histologic correlation, we utilized automatic, region-dependent thresholding that minimized errors related to signal heterogeneity. The algorithm will be further developed to include measures of CEP curvature and CEP damage fraction. Variations in CEP curvature have been shown to alter the distribution of disc loading, ${ }^{37}$ which may ultimately affect disc health.

Previous work has shown that CEP damage colocates with Modic lesions, ${ }^{18}$ which are also highly specific predictors of back pain. ${ }^{38,39}$ This may be due to factors secreted by nucleus cells that diffuse through damaged CEPs and irritate bone marrow cells. For example, osteoclastic activators are elevated in discs with Modic lesions, ${ }^{40}$ and coincident cellular behaviors of nucleus and marrow cells indicate detrimental disc/bone crosstalk. ${ }^{41}$ Future studies that use similar imaging approaches as we have described here may help delineate associations between CEP damage, Modic lesions, and physical symptoms. Given the strong association between CEP damage and back pain/injury, ${ }^{16-}$ ${ }^{19}$ we anticipate that standardized measures of CEP damage will add value to large cohort studies.

This study has several limitations. First, the resolution of the MR images limits the accuracy of CEP thickness measurements. Specifically, the in-plane resolution was $0.5 \times 0.5$ $\mathrm{mm}^{2}$, while the CEP thickness only ranged from 0 to $1.5 \mathrm{~mm}$ thick. The $0.5 \mathrm{~mm}$ voxel size resulted in partial volume averaging, which is a likely reason for the significant proportional bias $(-0.25 \pm 0.73 \mathrm{~mm})$. However, since the magnitude of the bias was small and did not depend on spinal level or Pfirrmann grade, the MRI estimates seem to reflect the thickness changes well. Furthermore, to help counteract the effects of limited resolution, we incorporated a resampling step in our algorithm that quadruples the number of pixels. This greatly improved our correlation with histologybased measures of CEP thickness. The negative proportional bias also indicates that UTE measurements underestimate CEP thickness for discs with thinner cartilage, which may 
make this technique less accurate for highly degenerated discs. Another limitation was that CEPs were sometimes difficult to segment near the edges of the disc due to decreased contrast between the annulus fibrosus and CEP, leading to overestimation of thickness. However, our correlation analysis between CEP thickness and disc $\mathrm{T}_{1 \rho}$ was conducted for the central disc, and thus possible errors in the peripheral CEPs did not affect our correlation analysis. Some specimen images had overall poor contrast, but this was overcome by the addition of the previously discussed threshold multiplier. Increased image quality in future studies may allow for removal of the threshold multiplier, making the algorithm fully automatic. Lastly, our small sample size was derived from adults with a narrow age range, and expansion to larger and younger cohorts is required to confirm some of the findings. For example, including a greater number of healthier discs may provide a wider range of T1rho values, which could improve the significance of the correlations between disc health and cartilage endplate morphology (Figure 7B and C).

In conclusion, our work demonstrates that quantitative measurements of the spatial variation of CEP thickness measured from UTE images are associated with disc degeneration. Spatial variations in CEP thickness and $T_{1 \rho}$ in the disc were strongly associated with Pfirrmann grade. These two parameters may serve as important quantitative and objective diagnostic markers for back pain patients.

\section{Key Points}

- Cartilage endplate thickness was measured from ultrashort echo-time MRI using a novel automatic segmentation algorithm.

$\square$ Variation in and $T_{1 \rho}$ was significantly lower for discs with higher Pfirrmann grades.

- Variation in cartilage endplate thickness was significantly correlated with variation in $T_{1 \rho}$.

- Our results suggest that variation in cartilage endplate thickness may be an important clinical marker of disc degeneration.

\section{References}

1. Andersson GB. Epidemiological features of chronic low-back pain. Lancet 1999;354:581-5.

2. Katz JN. Lumbar disc disorders and low-back pain: socioeconomic factors and consequences. J Bone Joint Surg Am 2006;88:21-4.

3. Hoy D, Bain C, Williams G, et al. A systematic review of the global prevalence of low back pain. Arthritis Rheum 2012;64:2028-37.

4. Luoma K, Riihimäki H, Luukkonen R, et al. Low back pain in relation to lumbar disc degeneration. Spine (Phila Pa 1976) 2000;25:487-92.

5. Peng B, Hou S, Wu W, et al. The pathogenesis and clinical significance of a high-intensity zone (HIZ) of lumbar intervertebral disc on MR imaging in the patient with discogenic low back pain. Eur Spine J 2006;15:583-7.

6. Raj PP. Intervertebral disc: anatomy-physiology-pathophysiologytreatment. Pain Pract 2008;8:18-44.
7. Roberts S, Menage J, Urban J. Biochemical and structural properties of the cartilage end-plate and its relation to the intervertebral disc. Spine (Phila Pa 1976) 1989;14:166-74.

8. Moore RJ. The vertebral end-plate: what do we know? Eur Spine J 2000;9:92-6.

9. Urban J, Holm S, Maroudas A, et al. Nutrition of the intervertebral disk: an in vivo study of solute transport. Clin Orthop Relat Res 1977;129:101-14.

10. Adams MA, Roughley PJ. What is intervertebral disc degeneration, and what causes it? Spine (Phila Pa 1976) 2006;31:2151-61.

11. Modic MT, Ross JS. Lumbar degenerative disk disease. Radiology 2007;245:43-61.

12. Martin MD, Boxell CM, Malone DG. Pathophysiology of lumbar disc degeneration: a review of the literature. Neurosurg Focus 2002;13:E1.

13. DeLucca JF, Cortes DH, Jacobs NT, et al. Human cartilage endplate permeability varies with degeneration and intervertebral disc site. J Biomech 2016;49:550-7.

14. Roberts S, Urban JP, Evans H, et al. Transport properties of the human cartilage endplate in relation to its composition and calcification. Spine (Phila Pa 1976) 1996;21:415-20.

15. Bernick S, Cailliet R. Vertebral end-plate changes with aging of human vertebrae. Spine (Phila Pa 1976) 1982;7:97-102.

16. Rajasekaran S, Bajaj N, Tubaki V, et al. ISSLS Prize winner: the anatomy of failure in lumbar disc herniation: an in vivo, multimodal, prospective study of 181 subjects. Spine (Phila Pa 1976) 2013;38:1491-500.

17. Lama P, Zehra U, Balkovec C, et al. Significance of cartilage endplate within herniated disc tissue. Eur Spine J 2014;23: 1869-77.

18. Fields AJ, Liebenberg EC, Lotz JC. Innervation of pathologies in the lumbar vertebral end plate and intervertebral disc. Spine J 2014;14:513-21.

19. Thompson KJ, Dagher AP, Eckel TS, et al. Modic changes on MR images as studied with provocative diskography: clinical relevance-a retrospective study of 2457 disks 1. Radiology 2009; 250:849-55.

20. Moon SM, Yoder JH, Wright AC, et al. Evaluation of intervertebral disc cartilaginous endplate structure using magnetic resonance imaging. Eur Spine J 2013;22:1820-8.

21. Bae WC, Statum S, Zhang Z, et al. Morphology of the cartilaginous endplates in human intervertebral disks with ultrashort echo time MR imaging. Radiology 2013;266:564-74.

22. DeLucca JF, Peloquin JM, Smith LJ, et al. MRI quantification of human spine cartilage endplate geometry: comparison with age, degeneration, level, and disc geometry. J Orthop Res 2016;34: 1410-7.

23. Fields AJ, Han M, Krug R, et al. Cartilaginous end plates: quantitative MR imaging with very short echo times-orientation dependence and correlation with biochemical composition. Radiology 2015;274:482-9.

24. Pfirrmann CW, Metzdorf A, Zanetti M, et al. Magnetic resonance classification of lumbar intervertebral disc degeneration. Spine (Phila Pa 1976) 2001;26:1873-8.

25. Weidenbaum M, Foster R, Best B, et al. Correlating magnetic resonance imaging with the biochemical content of the normal human intervertebral disc. J Orthop Res 1992;10:552-61.

26. Perry J, Haughton V, Anderson P, et al. The value of T2 relaxation times to characterize lumbar intervertebral disks: preliminary results. AM J Neuroradiol 2006;27:337-42.

27. Pandit P, Talbott JF, Pedoia V, et al. T1rho and T2-based characterization of regional variations in intervertebral discs to detect early degenerative changes. J Orthop Res 2016;34:1373-81.

28. Auerbach JD, Johannessen W, Borthakur A, et al. In vivo quantification of human lumbar disc degeneration using T1\rhoweighted magnetic resonance imaging. Eur Spine J 2006;15: $338-44$.

29. Blumenkrantz G, Zuo J, Li X, et al. In vivo 3.0-tesla magnetic resonance T1rho and T2 relaxation mapping in subjects with intervertebral disc degeneration and clinical symptoms. Magn Reson Med 2010;63:1193-200. 
30. Li X, Han ET, Busse RF, et al. In vivo T1rho mapping in cartilage using 3D magnetization-prepared angle-modulated partitioned $\mathrm{k}$ space spoiled gradient echo snapshots (3D MAPSS). Magn Reson Med 2008;59:298-307.

31. Lanczos C. Trigonometric interpolation of empirical and analytical functions. Stud Appl Math 1938;17:123-99.

32. Madhukar B, Narendra R. Lanczos resampling for the digital processing of remotely sensed images. Proceedings of International Conference on VLSI, Communication, Advanced Devices, Signals \& Systems and Networking 2013:403-411.

33. Otsu N. A threshold selection method from gray-level histograms. IEEE Trans Syst Man Cybern Syst 1979;9:62-6.

34. Carballido-Gamio J, Bauer JS, Lee KY, et al. Combined image processing techniques for characterization of MRI cartilage of the knee. Conf Proc IEEE Eng Med Biol Soc 2005;3:3043-6.

35. Nachemson A, Lewin T, Maroudas A, et al. In vitro diffusion of dye through the end-plates and the annulus fibrosus of human lumbar inter-vertebral discs. Acta Orthop Scand 1970;41:589-607.
36. Setton LA, Zhu W, Weidenbaum M, et al. Compressive properties of the cartilaginous end-plate of the baboon lumbar spine. $J$ Orthopaed Res 1993;11:228-39.

37. Langrana NA, Kale SP, Edwards WT, et al. Measurement and analyses of the effects of adjacent end plate curvatures on vertebral stresses. Spine J 2006;6:267-78.

38. Weiner BK, Vilendecic M, Ledic D, et al. Endplate changes following discectomy: natural history and associations between imaging and clinical data. Eur Spine J 2015;24:2449-57.

39. Dudli S, Fields AJ, Samartzis D, et al. Pathobiology of Modic changes. Eur Spine J 2016;25:3723-34.

40. Torkki M, Majuri ML, Wolff $\mathrm{H}$, et al. Osteoclast activators are elevated in intervertebral disks with Modic changes among patients operated for herniated nucleus pulposus. Eur Spine J 2016;25:207-16.

41. Dudli S, Sing DC, Hu SS, et al. ISSLS PRIZE IN BASIC SCIENCE 2017: intervertebral disc/bone marrow cross-talk with Modic changes. Eur Spine J 2017;26:1362-73. 